\title{
Evaluación del seguimiento de 29 niños chilenos con la enfermedad de la orina olor a jarabe de arce clásica
}

\author{
Follow up evaluation of 29 \\ chilean children with classical \\ maple syrup urine disease
}

\begin{abstract}
Introduction: Maple Syrup Urine Disease (MSUD) is caused by a defect of the ketoacid dehydrogenase enzyme complex of the branched amino acids Valine, Isoleucine and Leucine (VIL). The treatment consists of a leucine-restricted diet. Objective: To evaluate the long-term follow-up in children with MSUD. Methodology: 29 records were reviewed of patients with MSUD, of which 24 were clinically identified (> 5th day of life), 4 cases by MSUD family history and one by neonatal screening ( $<5$ th day of life). Leucine (Leu) levels were measured at diagnosis (Biotronic 2000) and during follow-up (mass spectrometry). The number of decompensation events, Total Intellectual Quotient (TIQ, Bayley and Wechsler scale) and nutritional status were also measured. STATA statistical software version 9.2 was applied ( $p \leq 0.05$ ). Results: Mean age at diagnosis was 14 days old. In all cases the diagnosis was confirmed by elevated levels of Leu and alloisoleucin. When comparing the TIQ of 19 cases over 3 years old with their age at diagnosis, it was observed that those cases screened by the 5th day of life had a TIQ $84.6 \pm 13$, while those diagnosed later had a TIQ $73 \pm 17$ ( $p \leq 0.05)$. In assessing the number of hospitalizations that occurred during follow-up, we determined that the 5 cases screened early never had a metabolic crisis and had a higher TIQ than those who had had one or more decompensation (92 and 74, respectively, $p \leq 0.05$ ). An inverse correlation was observed between the Leu+Isoleucine value and TIQ. Conclusion: The diagnosis before the 5th day of life and a good metabolic control during follow-up, enables children with MSUD to have normal cognitive development.
\end{abstract}

Key words: Maple syrup urine disease, leucine, intelligent quotient.
(1) Laboratorio de Genética y Enfermedades Metabólicas, Instituto de Nutrición y Tecnología de los Alimentos (INTA), Universidad de Chile, Santiago, Chile.

(2) Laboratorio de Enfermedades Metabólicas, Hospital Carlos Van Buren, Valparaíso.

$$
\begin{array}{r}
\text { Dirigir la correspondencia a: } \\
\text { Profesora } \\
\text { Verónica Cornejo E } \\
\text { LabGEM } \\
\text { INTA, Universidad de Chile } \\
\text { Casilla 13811, Santiago } \\
\text { Chile } \\
\text { Fax 56-2-2941254 } \\
\text { E-mail: vcornejo@inta.uchile. cl }
\end{array}
$$

Este trabajo fue recibido el 4 de Marzo de 2014 y aceptado para ser publicado el 24 de Junio de 2014.

\section{INTRODUCCIÓN}

La enfermedad de la Orina Olor a Jarabe de Arce (EOJA) (OMIM 248600), es de herencia autosómica recesiva causada por un defecto del complejo enzimático deshidrogenasa de los aminoácidos ramificados: Valina, Isoleucina y Leucina (VIL) y del cetoácido alfa cetoisocaproico proveniente de la Leucina (Leu) (1). Esta acumulación ocasiona un compromiso neurológico que puede llevar a la muerte o dejar graves secuelas neurológicas, si no se diagnostica precozmente y no se trata adecuadamente a largo plazo $(2,3)$.

La forma clásica se presenta en recién nacidos de apariencia normal y se manifiesta con signos y síntomas poco específicos al término de la primera semana de vida, tales como vómito letargia, irritabilidad, baja succión. Sin tratamiento se produce un deterioro neurológico progresivo que puede producir un retardo mental irreversible o llevar a la muerte. Junto a estos síntomas hay un olor característico a azúcar quemada o jarabe de arce en piel y orina (4). El diagnóstico se confirma por los niveles elevados de VIL por la presencia del ácido orgánico alloisoleucina, considerado patognomónico en esta patología.

La frecuencia mundial descrita a través de los programas de pesquisa neonatal es de aproximadamente 1:185 recién nacidos (5). En Chile aún no se ha implementado la pesquisa 
en el recién nacido, por tanto todo los casos diagnosticados, se han establecido por la descompensación metabólica o por antecedentes familiares con EOJA.

El tratamiento consiste en una dieta restringida en Leu que prohíbe el consumo de alimentos de origen animal (carnes en general, lácteos y derivados, pescados y marisco, legumbres), con una ingesta controlada de cereales, verduras y frutas, siendo obligatorio el aporte de una fórmula láctea sin VIL como única fuente de proteínas de alto valor biológico (6). Conjuntamente deben ser suplementados con tiamina (50-100 mg/d) para estimular la actividad enzimática residual y con los aminoácido libres L-Val y L-Iso, los que compiten con la Leu por el mismo transportador para cruzar la barrera hematoencefálica (7). El propósito del tratamiento es evitar el catabolismo, mantener el valor de Leu en suero bajo 200 $\mu M$ y prevenir las secuelas neurológicas que esta patología ocasiona en cualquier momento de la vida (8).

Se ha demostrado que un diagnóstico durante los primeros días de vida y la buena adherencia al tratamiento nutricional a largo plazo, favorece el pronóstico de esta enfermedad $(9,10)$.

El Instituto de Nutrición y Tecnología de los Alimentos (INTA), de la Universidad de Chile, ha diagnosticado 40 niños con EOJA clásica, de los cuales 29 casos se mantienen en control metabólico.

El objetivo de esta publicación fue evaluar el seguimiento a largo plazo y sus implicancias en el crecimiento y desarrollo.

\section{METODOLOGÍA}

Es un estudio retrospectivo y se revisaron 40 fichas de los pacientes con EOJA diagnosticados entre los años 1989 y 2009 por el INTA, único centro en Chile que realiza la confirmación diagnóstica y cuenta con un equipo médico especializado que realiza el seguimiento de los casos. Los criterios de inclusión fueron: estar en seguimiento y no tener otras patologías asociadas. Dos casos fueron excluidos por estar sin seguimiento y 9 casos fallecieron durante el primer mes de vida, en la fase aguda debido a la grave descompensación metabólica.

El grupo estudio quedó conformado por 29 niños con EOJA clásica (16 sexo femenino y 13 masculino). La media de edad al diagnóstico fue 14 días de vida con un rango intercuatílico (RIQ) de 6. Del total de la muestra, 24 casos fueron diagnosticados después del 5 to día de vida y 5 casos antes (4 niños tenían un hermano con EOJA, y un caso fue detectado a través del programa de pesquisa neonatal proveniente de una clínica privada de San Fernando VI región). El rango de edad actual fluctuó entre los 1.6 y los 20.6 años de edad, con un promedio de 10,5 años de seguimiento en el INTA.

Evaluación del estado nutricional: todos los pacientes fueron pesados en una balanza (Seca) con una exactitud de 0,05 k, y medidos en un tallimetro con un margen de error de $0.01 \mathrm{~cm}$, actividades realizadas por el equipo de salud tratante, usando métodos debidamente estandarizados. Se calculó en menores de un año el peso/edad, entre el 1 y los 6 año se utilizó el peso/ talla y en los mayores de seis años el Índice de Masa Corporal (IMC (kg/m2). Para clasificar el estado nutricional se usó como patrón de referencia las normas del Ministerio de Salud según edad y sexo de la OMS 2007 (11 - 13).

Ingesta nutricional: se obtuvo de la encuesta nutricional de los 3 últimos controles registrados en la ficha clínica. Se calculó la ingesta de Leu a través del programa computacional aminoacid analyzer (AAA) y los resultados fueron comparados con las recomendaciones para niños con EOJA. Se consideró una adecuación nutricional normal cuando estaba entre el $90 \%$ a $110 \%$, déficit cuando era menor al $90 \%$ y exceso cuando era mayor al $110 \%$ (14).

Medición de niveles de aminoácidos: la confirmación diagnóstica se realizó a través de la cuantificaron de VIL en suero (15) (Biotronic 2000, Hamburgo, Alemania) y se compararon con los valores de referencia normales (Val 64-294 $\mu$ M, Iso 31-86 $\mu M$ y Leu 47-155 $\mu M$ ). Se consideró buen control metabólico cuando el valor de Leu en suero estaba bajo 200 $\mu M$. Los valores de VIL durante el seguimiento se midieron a través de la técnica de espectrometría de masa en Tándem (MS/MS) (MicroMass, Waters Corporation, Mildford, USA) (16) en sangre seca recogida en tarjeta de papel filtro, usando reactivos comerciales (Cambridge Isotope Laboratories) (17). La técnica MS/MS cuantifica los aminoácido Leu+lso en el mismo pico, porque son moléculas isobáricas (VN:10-200 $\mu \mathrm{M}$ ) y la Val independientemente (VN:43-243 $\mu \mathrm{M})$. El valor bajo 300 uM de Leu+Iso obtenido de sangre total, es considerado un buen control metabólico.

Evaluación psicométrica: se utilizó la escala de desarrollo infantil de Bayley en menores de 36 meses (Índice de Desarrollo Mental y Motor (MDI/PDI): normal 85-115, riesgo 70-84 y retraso significativo $<69$ ) (18) y la prueba de inteligencia infantil de Wechsler en los mayores de 3 años (Coeficiente de Inteligencia total (CIT): normal 80 110, limítrofe 70-79, retraso mental leve 55-69) (19). El grupo estudio fue evaluado por la escala de desarrollo correspondiente a su edad y de acuerdo a estos resultados se realizó análisis estadístico, separando los menores de 36 meses y los mayores de 3 años. Las pruebas fueron aplicadas por la psicóloga del programa de seguimiento del INTA.

Descompensación metabólica: se clasificó como descompensación a todas las hospitalizaciones ocasionadas por valores de Leu sobre 600 uM, con compromiso de conciencia o ataxia.

Análisis estadístico: Se aplicó programa estadístico STATA versión 9.2 para Windows (StataCorp LP, College Station, Tx, USA). Se fijó la significancia estadística con un valor de $p \leq 0.05$. Se verificó la distribución normal de las variables (Prueba Shapiro Wilk) y para determinar diferencias entre grupos se usó la prueba t-Student, para asociación de variables categóricas y para determinar variables predictivas del desarrollo cognitivo se utilizó la correlación de Pearson y regresión lineal. Aquellas variables cuya distribución no era normal, se aplicó pruebas no paramétricas (Wilcoxon Mann-Whittney y correlación de Spearman). Los datos fueron presentados en rangos, y/o media, mas desviaciones estándar.

\section{RESULTADOS}

Lo signos y síntomas clínicos encontrados con mayor frecuencia en los 24 casos diagnosticados por descompensación metabólicas fueron: olor a jarabe de arce (21/29), somnolencia (21/29), irritabilidad (16/29), hipertonía (16/29) e hipotonía (15/29) y la mediana del los niveles de VIL en suero fueron: Leu $1930 \mu M(R I Q=1236)$, Val $506 \mu M(R I Q=377)$ e Iso 457 $\mu M(R I Q=323)$. Los 5 casos diagnosticados antes del 5 to día de vida, nunca tuvieron crisis metabólica y la mediana al diagnóstico de Leu fueron de $845 \mu M(R I Q=360)$, Iso de $196 \mu M$ ( $R I Q=34)$ y Val de $549 \mu M(R I Q=427)$, siendo esta diferencia significativas (figura 1).

El desarrollo infantil (escala de Bayley) de 9 casos fue clasificado como retraso significativo y en un caso como normal. EI CIT (escala de Whechsler) en los 19 casos restantes fue catalogado en 6/29 como retraso mental leve, 6/29 eran limítrofes y $7 / 29$ tuvieron un CIT normal. 
Al relacionar el nivel de Leu sérica registrada al diagnóstico con el coeficiente CIT obtenido durante el seguimiento, se encontró una asociación significativa $(p \leq 0.05)$. Los niños que tuvieron valores de Leu sobre $1000 \mu \mathrm{M}$, presentaron un CIT de 72,07 y los niños con valores de Leu inferiores a 1000 $\mu M$, tuvieron un CIT de 87,2 ( $p \leq 0.05$ ) (figura 2 ).

Al separar por edad de diagnóstico los 19 casos mayores de 3 años y determinar el CIT obtenido durante el seguimiento, se pudo observar que aquellos casos pesquisados antes del 5 to día tenían un CIT mayor que los diagnosticados posteriormente

\section{FIGURA 1}

Comparación entre el nivel de leucina inicial y edad de diagnóstico en 29 niños con EOJA clásicos.

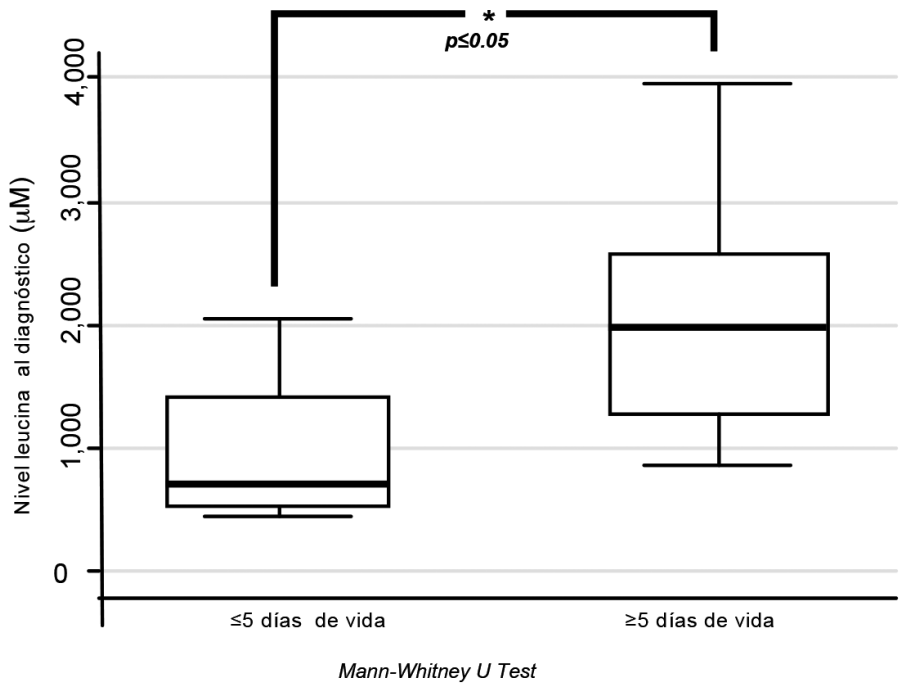

FIGURA 2

Coeficiente intelectual según nivel plasmático de leucina al diagnóstico en 19 niños con EOJA mayores de 3 años.

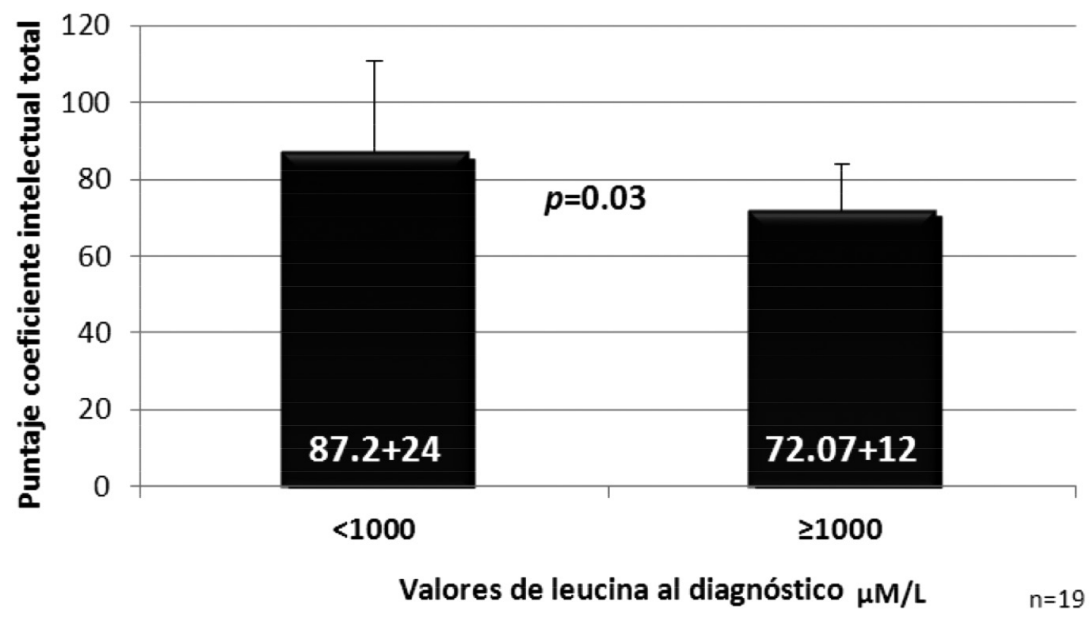


(CIT $=84,6 \pm 13$ y $73 \pm 17$ respectivamente), no obstante no hubo diferencias significativas (figura 3 ).

Considerando que esta patología causa descompensaciones graves durante el seguimiento ocasionadas principalmente por cuadros infecciosos agudos o transgresiones alimentarias, se registró el número de hospitalizaciones durante el seguimiento; observándose que aquellos que nunca tuvieron una crisis metabólica, tenían una mediana del CIT de 92 y los que habían tenido una o más descompensaciones tuvieron un CIT de 74, siendo estas diferencias significativa ( $p \leq 0.05$ ) (figura 4).

FIGURA 3

Coeficiente intelectual según edad de diagnóstico en 19 niños con EOJA clásicos.

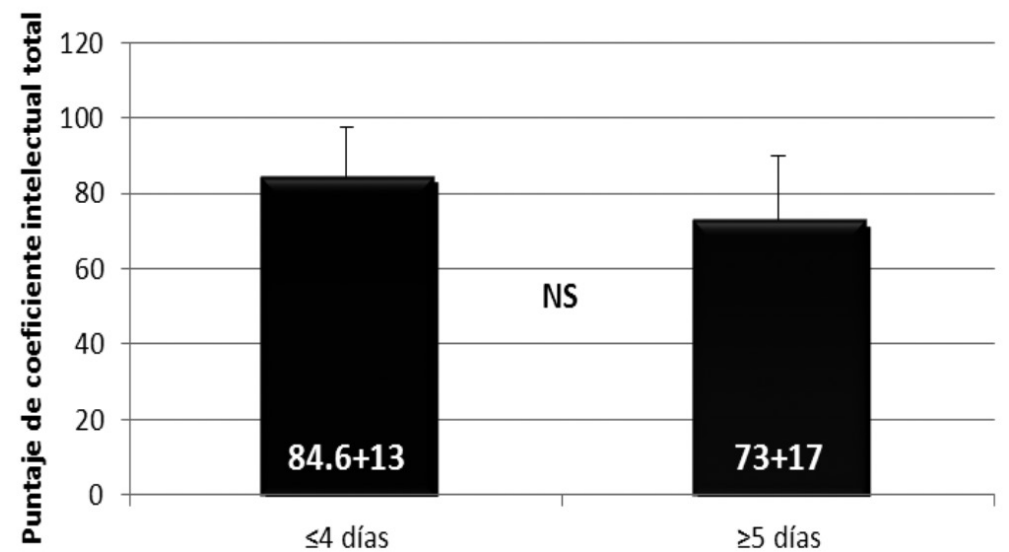

Edad de diagnóstico (días)

$n=19$

$\left(\chi^{2}\right.$ Pearson 0.5435, $\left.\mathrm{p}=0.461\right)$

FIGURA 4

Coeficiente intelectual según presencia o ausencia de descompensación metabólica en niños con EOJA.

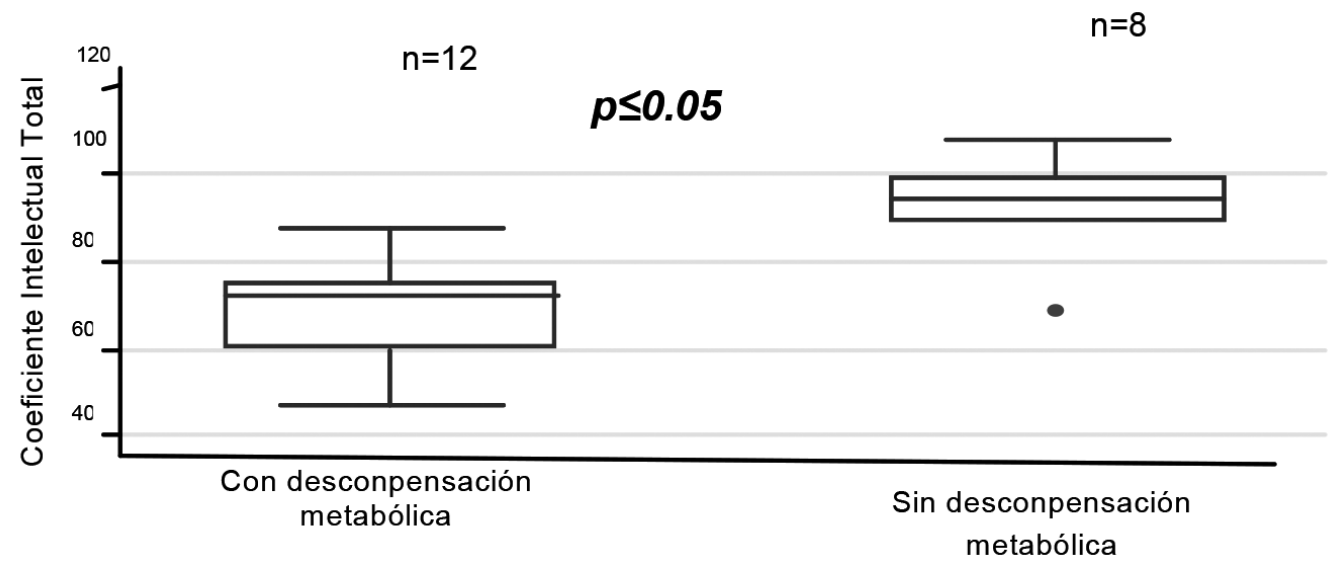


Detectándose además una correlación inversa entre el número de descompensaciones y el CIT (figura 5).

También se encontró diferencias significativas cuando se comparó el valor Leu+Iso mantenido durante el seguimiento entre los casos que nunca tuvieron una descompensación con los habían tenido una o más ( $\mathrm{p} \leq 0.05)$ (figura 6). Al correlacionar este valor de Leu+lso con el CIT, se observó una relación inversamente proporcional, interpretándose que valores elevado de Leu+lso mantenidos durante el seguimiento, disminuye el CIT del niño con EOJA (figura 7).

\section{FIGURA 5}

Relación entre coeficiente intelectual y numero de descompensaciones en niños con EOJA clásico en seguimiento.

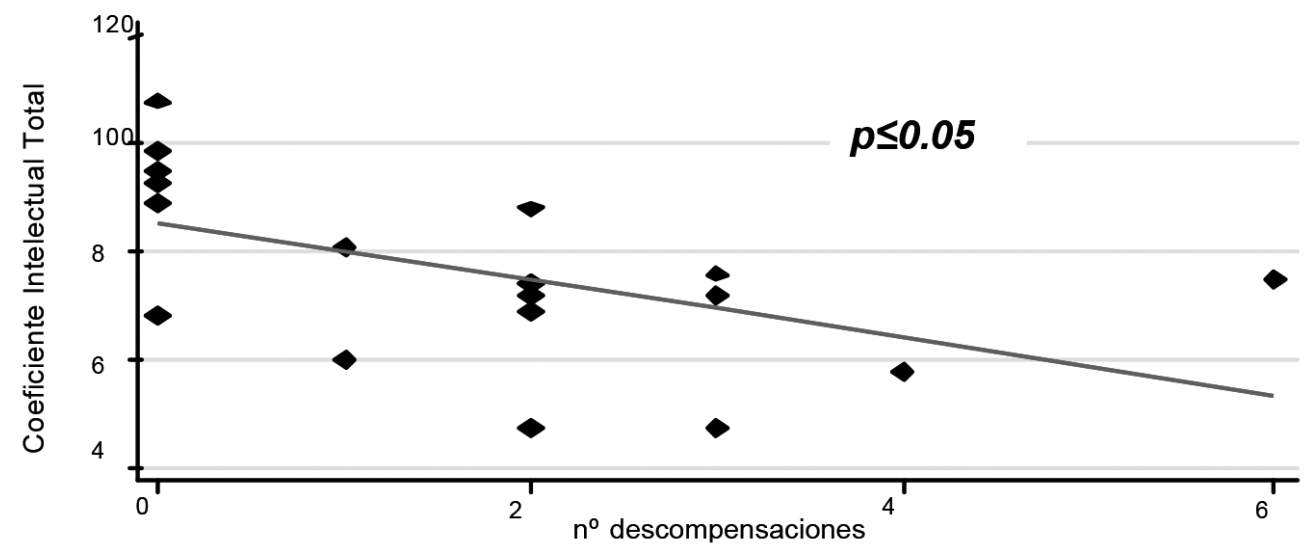

Correlación de Spearman

\section{FIGURA 6}

Valor promedio de Leu+lso de seguimiento y presencia o ausencia de descompensación metabólica en 29 niños con EOJA clásica.

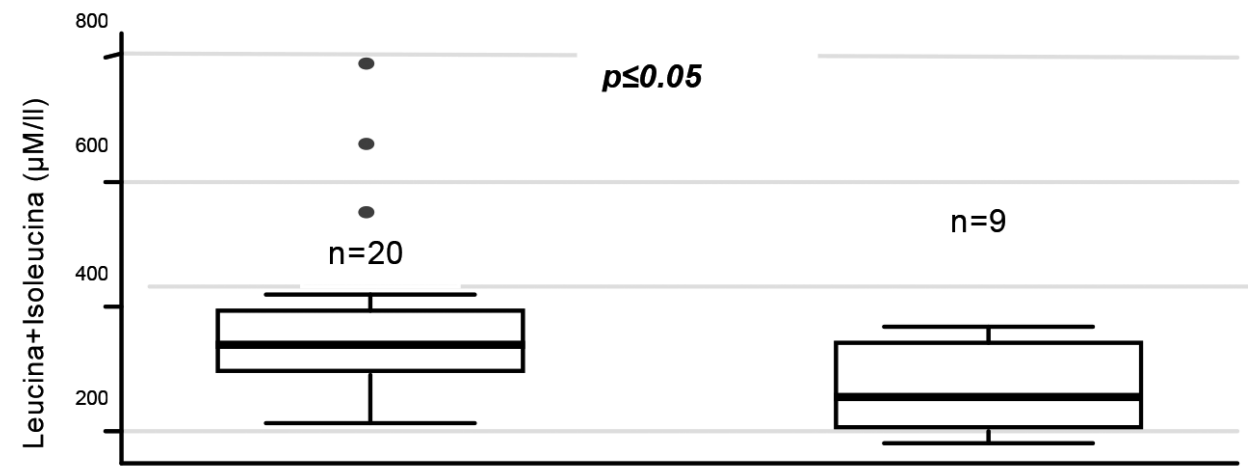

con descompensación metabólica sin descompensación

metabólica 
Considerando esto un hallazgo, se aplicó un análisis multivariado para determinar si existía una influencia del valor de Leu+Iso sobre el CIT, considerándose las variables: edad y valor de Leu al diagnóstico, estado nutricional, número de descompensaciones durante el seguimiento, modelo estadístico que sólo explicó el 55,9\% de la variabilidad del $\mathrm{Cl}(\mathrm{p}=0,02)$, siendo el valor de Leu+lso la única variable predictiva $(p=0,025)$.

En relación a la ingesta del aminoácido Leu, se encontró que los menores de 18 años mantenían una ingesta de acuerdo a las recomendaciones, pero los mayores de 18 años (3/29) tenían una adecuación del 125\% por sobre lo recomendado (tabla 1).

De acuerdo a la evaluación del estado nutricional se encontró que 12 niños tenían talla baja para la edad, 13 eran eutróficos y 6 niños tenían riesgo de obesidad. Los 3 casos mayores de 18 años tuvieron un IMC normal.

\section{DISCUSIÓN}

Un estudio de Filipina mostró que los síntomas clínicos aparecieron en promedio al 5to día de vida, pero el diagnóstico fue establecido a los 39 días de vida (20). En nuestra casuística los signos clínicos se manifestaron desde el 5 to día de vida,

\section{FIGURA 7}

Relación entre valor de Leu+lso durante el seguimiento y coeficiente intelectual en 19 niños con EOJA clásica.

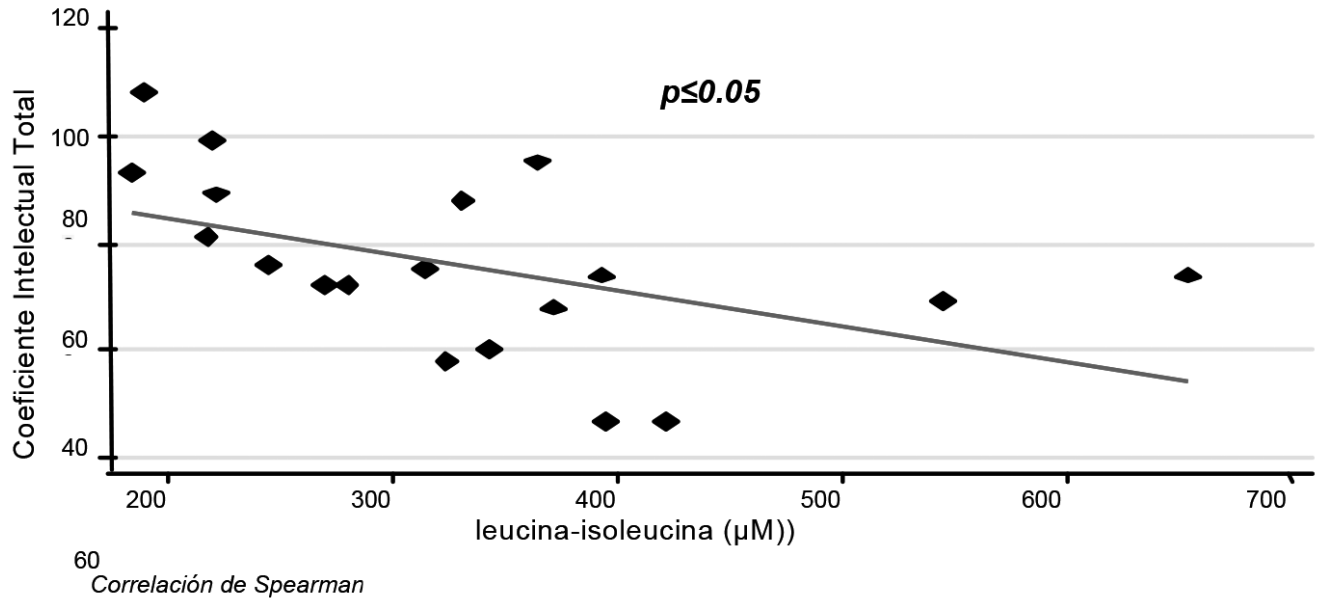

TABLA 1

Ingesta promedio y adecuación de leucina según recomendaciones para EOJA clásica por rangos de edad.

\begin{tabular}{cccc}
\hline $\begin{array}{c}\text { Rango de edad } \\
\text { (años) }\end{array}$ & $\begin{array}{c}\text { Ingesta leucina total } \\
(\mathrm{mg} / \text { día })\end{array}$ & $\begin{array}{c}\text { Recomendación } \\
\text { Ingesta leucina } \\
\text { (mg/día) }\end{array}$ & $\begin{array}{c}\text { Adecuación } \\
(\%)\end{array}$ \\
$1-4$ & $350(213)$ & $275-535$ & $90-110$ \\
$4,1-7$ & $512(124)$ & $360-695$ & $90-110$ \\
$7,1-11$ & $563(168)$ & $410-785$ & $90-110$ \\
$11,1-15$ & $562(202,5)$ & $50-740$ & $90-110$ \\
Mujeres & $424,5(7,5)$ & $540-720$ & $90-110$ \\
Hombres & & & 200 \\
$>18$ & 1237 & $400-620$ & 125 \\
Mujeres & $1381(581)$ & $800-1100$ & \\
Hombres & & & \\
\hline
\end{tabular}


pero el diagnóstico se logró establecer a los 14 días de vida, mucho antes de lo reportado en ese estudio. Esto nos permite especular que el equipo de salud de Chile frente a un niño agudamente enfermo, sospecha en primera instancia en una enfermedad metabólica.

Cuando se relaciona el nivel de Leu de inicio con la edad de diagnóstico, diversos estudios han señalado que existe una relación inversa, ya que mientras más tarde se confirme la patología, el valor de Leu se incrementa, debido a los procesos catabólicos asociados al nacimiento y al ayuno ocasionado por la crisis encefalopática; efecto que tiene una influencia negativa sobre el desarrollo cognitivo e intelectual (21 - 23). Este estudio encontró diferencias significativa entre estas variables, observándose que los pacientes con EOJA detectados antes del 5 to día de vida tuvieron niveles de Leu 2 veces más bajo que los diagnosticados posteriormente y el CIT obtenido a largo plazo fue de $84.6 \pm 13$, un desarrollo intelectual 11 puntos por sobre lo obtenido en los casos pesquisados después del 5 to día de vida $(73 \pm 17)$.

En un estudio realizado en la cohorte de los Menonitas encontró que los CIT eran diferentes entre el grupo control y Ios EOJA en tratamiento (CIT $106 \pm 15$ y $81 \pm 19$ respectivamente), pero sin diferencias significativa (24). Al comparar el CIT entre un grupo de EOJA en dieta y un grupo con EOJA con trasplante hepático, encontraron una correlación inversa entre la edad de diagnóstico y el CIT (24). Si bien nuestro estudio no encontró una diferencia significativa entre la edad de diagnóstico y el CIT, lo que podría atribuirse a que el tamaño de la muestra de este estudio fue insuficiente para determinar diferencias. Sin embargo, cuando se comparó el CIT de los niños diagnosticados antes y después del 5 to día de vida, y este valor fue clasificado e interpretado de acuerdo al puntaje de CIT obtenido, se observó que aquellos niños diagnosticados antes del 5 to día de vida que tenían un CIT de 84,6 clasificado como normal, en comparación con los casos diagnósticados posteriormente cuyo puntaje fue de 73,17 , resultado que fue catalogado como limítrofe. Basándonos en estos resultados absolutos, se podría deducir que la edad de diagnóstico influye en el pronóstico del desarrollo cognitivo a largo plazo, pero esta aseveración para validarse estadísticamente, requiere de otro estudio que incluya un mayor número de casos que permita verificar este hallazgo.

No obstante el diagnóstico temprano no es el único factor que permite un desarrollo cognitivo dentro de rangos de normalidad, sino que hay otros factores durante el seguimiento que también influyen el CIT. En nuestro estudio se demostró que el valor obtenido de Leu+lso durante el seguimiento, es la variable de mayor influencia sobre el CIT, lo que nos permite deducir que los niños con buen control metabólico, tienen un mejor desarrollo cognitivo.

Un punto importante a discutir es la importancia de la fórmula sin VIL, que en el tratamiento de esta patología es muy importante para lograr un buen control metabólico y obtener un crecimiento en rangos de normalidad. En nuestro estudio hubo 12 casos con talla baja, debido a una menor ingesta de proteínas de alto valor biológico, producto al bajo consumo de fórmulas especial sin VIL. Esto se debido a que durante el periodo que se realizó este estudio, el Estado no subvencionaba la fórmula especial y era la familia quien debía de asumir el costo de producto, que en la mayoría de las veces no lograba cubrir las recomendaciones de este nutriente esencial para el crecimiento, por el elevado costo. Sin embargo se debe enfatizar que desde el año 2012, el Ministerio de Salud entrega gratuitamente y de forma Universal las fórmulas especiales sin
VIL a todos los niños con esta patología.

Con estos resultados podemos concluir que es imperativo iniciar el diagnóstico neonatal de EOJA en Chile, ya que un niño con una EOJA clásica diagnosticado antes del 5to día de vida, y que mantenga un buen control metabólico durante el seguimiento, logran tener un crecimiento y desarrollo en rangos de normalidad.

\section{RESUMEN}

La enfermedad de la orina olor a jarabe de arce (EOJA) se produce por un defecto del complejo enzimático deshidrogenasa de los cetoácidos de los aminoácidos ramificados: Valina, Isoleucina, Leucina (VIL). El tratamiento es una dieta restringida en leucina (Leu). Objetivo: evaluar el seguimiento a largo plazo en niños con EOJA. Metodología: Se revisaron 29 fichas de pacientes EOJA, 24 fueron pesquisados por clínica ( $>5$ to día de vida) y 4 casos por antecedentes familiares con EOJA y 1 por pesquisa neonatal ( $<5$ to día de vida). Se midió nivel de Leu al diagnóstico (Biotronic 2000) y durante el seguimiento (Espectrometría de masa), número de descompensaciones, Coeficiente Intelectual Total (CIT) (Escalas de Bayley y Wechsler) y estado nutricional. Se aplicó programa estadístico STATA versión 9.2 ( $p \leq 0.05)$. Resultados: La edad de diagnóstico fue a los 14 días de edad. En todos se confirmó el diagnóstico por los niveles elevados de Leu y presencia de alloisoleucina. Al comparar el CIT de los 19 casos mayores de 3 años con la edad de diagnóstico, se observó que aquellos casos pesquisados antes del 5to día tenían un CIT de 84,6 $6 \pm 13$, a diferencia de los diagnosticados posteriormente que tenían un $\mathrm{CIT}=73 \pm 17$ ( $\mathrm{p} \leq 0.05)$. Al evaluar el número de descompensaciones ocurridas durante el seguimiento, se determinó que los 5 casos nunca habían tenido una crisis metabólica, tuvieron un $\mathrm{Cl}$ mayor que aquellos que habían tenido una o más descompensaciones (92 y 74 respectivamente) ( $p \leq 0.05)$. Cuando se correlacionó el valor de Leu+lso de seguimiento con el CIT, se observó una correlación inversamente proporcional. Conclusión: el diagnóstico antes de los 5to día de vida y un buen control metabólico durante el seguimiento, permite que los niños con EOJA tengan un desarrollo cognitivo normal.

Palabras clave: Enfermedad de la orina olor a jarabe de arce, leucina, coeficiente Intelectual.

\section{BIBLIOGRAFÍA}

1. Morton DH, Strauss K, Robinson D, Puffennberger E, Kelley $R$. Diagnosis and treatment of maple syrup disease: A study of 36 patients. Pediatrics 2002; 106:999-1008.

2. Cornejo $V$, Raimann E. Errores innatos del metabolismo de los aminoácidos. En: Colombo M, Cornejo V, Raimann $E$, editores. Errores innatos en el metabolismo del niño. Santiago, Chile: Editorial Universitaria. 2010: p 65-87.

3. Zinnanti WJ, Lazovic J. Interrupting the mechanisms of brain injury in a model of maple syrup urine disease encephalopathy. J Inherit Metab Dis. 2012;35(1):71-9.

4. Jeong JS, Sim HJ, Lee YM, Yoon HR, Kwon HJ, Hong SP. Chromatographic diagnosis of maple syrup urine disease by measuring the L-alloisoleucine/L-phenylalanine ratio in dried blood spots. J Chromatogr B Analyt Technol Biomed Life Sci. 2011,15;879:2171-4.

5. Chuang DT, Shih VE. Maple syrup urine disease (branchedchain ketoaciduria). In: Scriver $C R$, Beaudet $A L$, Sly WS, Valle D, eds; Childs B, Kinzler KW, Vogelstein B, Assoc. eds. Metabolic Molecular Bases Inherited Dis, 8th Ed. New York: McGraw-Hill, 1971-2006. 2001.

6. Strauss KA1, Wardley B, Robinson D, Hendrickson C, Rider 
NL, Puffenberger EG, Shellmer D, Moser AB, Morton DH. Classical maple syrup urine disease and brain development: principles of management and formula design. Mol Genet Metab. 2010,99(4):333-45.

7. Knerr I, Weinhold N, Vockley J, Gibson KM. Advances and challenges in the treatment of branched-chain amino/keto acid metabolic defects. J Inherit Metab Dis. 2012;35(1):29-40.

8. Klee D, Thimm E, Wittsack HJ, Schubert D, Primke $R$, Pentang G, Schaper J, Mödder U, Antoch A, Wendel U, Cohnen $M$. Structural white matter changes in adolescents and young adults with maple syrup urine disease. J Inherit Metab Dis. 2013;36(6):945-53.

9. Serra D, Fernández-Sánchez A, Sánchez-Valverde F, Miñana 1. Enfermedad de la orina olor a jarabe de arce. En Sanjurjo $P$, Baldellou A, editores. Diagnóstico y tratamiento de las enfermedades metabólicas hereditarias, capítulo 35. Editorial Ergon SA, Madrid, España, 2010, 487-523.

10. Levy HL. Newborn screening conditions: What we know, what we do not know, and how we will know it. Genet Med. 2010;12(12 Suppl):S213-4.

11. Referencia OMS para la evaluación antropométrica para niñas y niños menores de 6 años. 2006. http://web.minsal.cl/ portal/url/item/e105731bee21b7a9e0400101650114c4. $p d f$

12. World Health Organization. WHO Child Growth Standards. 2007. http://www.who.int/childgrowth/en/

13. Unidad de Nutrición del Ministerio de Salud. Consejo Asesor en Nutrición. Norma técnica de evaluación nutricional del niño de 6 a 18 años. año 2003. Rev Chil Nutr [online]. 2004, 31(2):128-137 [citado 25-022014]. Disponible en: http://dx.doi.org/10.4067/S071775182004000200007

14. Acosta P. Maple Syrup Urine Disease (MSUD), Protocol 5. En: The Ross metabolic Formula System. Nutrition Support Protocols, 4th edition. Ohio, USA. Editorial Ross Products Division 2001:p 74-102.

15. Couce $M$, Castiñeiras D, Bóveda $M$, Iglesias A, Cocho De
Juan J, Fraga J. Avances en el diagnóstico y tratamiento de la enfermedad de jarabe de arce, experiencia en Galicia. Ann Pediatr. 2007; 67:337-43.

16. Schulze A, Linder M, Kohlmüller D, Olgemöller K, Mayatepek E, Hoffmann G. Expanded Newborn Screening for Inborn Errors of Metabolism by electrospray ionization - Tandem Mass Spectrometry: Results, outcome, and implications. Pediatrics 2003; 111:1399-406.

17. Cambridge Isotopes Laboratories Inc. http://www.isotope. $\mathrm{com} / \mathrm{cil} /$ products/listproducts.cfm?cat_id=72\&market=clin ical\&prodtypeid $=58$

18. Bayley N. 24 Months Bayley scales of infant development (MDI) En: Bayley N. Bayley Scales of mental and motor development. Editorial, The Psychological Corporation, Harcourt Brace Jovanovich, New York, 1969.ca

19. Ramírez V, Rosas R. Estandarización del WISC-III en Chile: Descripción del Test, Estructura Factorial y Consistencia Interna de las Escalas. Psykhe 2007;16:91-109.

20. Lee JY, Chiong MA, Estrada SC Cutiongco-De la Paz EM, Silao CL, Padilla CD. Maple syrup urine disease (MSUD)clinical profile of 47 Filipino patients. J Inherit Metab Dis. 2008;31 Suppl 2:S281-5.

21. Simon E, Figerhut $R$, Bramkötter J, Konstantopoulou $V$, Ratschmann $R$, Wendel $U$. Maple syrup urine disease: Favourable effect of early diagnosis by newborn screening on the neonatal course of de disease. J Inherit Metab Dis. 2006; 29:532-7.

22. Kaplan P1, Mazur A, Field M, Berlin JA, Berry GT, Heidenreich $R$, Yudkoff $M$, Segal S.Intellectual outcome in children with maple syrup urine disease. J Pediatr. 1991;119: 46-50.

23. Hilliges $C$, Awiszus D, Wendel U . Intellectual performance of children with maple syrup urine disease. Eur J Pediatr. 1993;152: 144-7.

24. Muelly ER1, Moore GJ, Bunce SC, Mack J, Bigler $D C$, Morton DH, Strauss KA. Biochemical correlates of neuropsychiatric illness in maple syrup urine disease. J Clin Invest 2013;123(4):1809-20. 